\title{
EXPLORACIONES GRÁFICAS DE IDEAS EXTRAESCOLARES DE LOS ALUMNOS SOBRE RADIACTIVIDAD
}

\author{
DE POSADA APARICIO, J.M. y PRIETo RUZ, T. \\ I. B. de Nerja (Málaga). \\ Departamento de Didáctica de las Ciencias Experimentales. Universidad de Málaga.
}

\begin{abstract}
SUMMARY
We present some aspects of radioactivity which somehow are familiar to the students, because as they pick information from outside the school, their level of Knowledge of pupils is not as low as one might expect.
\end{abstract}

\section{INTRODUCCIÓN}

Para Solomon (1988), los conceptos se forman en las mentes de los alumnos atendiendo a cuatro vías bien diferenciadas, la primera de ellas haciendo uso de constructos individuales mediante los cuales los alumnos intentan encontrar explicaciones a sus experiencias personales, en segundo lugar, los alumnos tienen contactos a veces esporádicos, a veces intensos, con los conceptos en el currículo, como consecuencia pueden superar, mal interpretar, adaptar, etc., dichas concepciones (Gilbert et al. 1982), por otro lado, el lenguaje y la cultura de la sociedad en la que vive el alumno, le induce unas determinadas tendencias, en último lugar, el conocimiento cotidiano (procedentes de charlas entre amigos, television, radio, periódicos y semanarios, etc.) se ve reforzado sobre el conocimiento científico debido al continuo contacto, flujo y reflujo de ideas que a veces equivocadas, erróneas o incompletas, dichos medios propagan.

Los trabajos previos encontrados sobre la radiactividad hacen mención a los aspectos psicológicos que se derivan de las actitudes y reacciones emocionales, que lo nuclear despierta en jovenes norteamericanos de doce a dieciocho años (Tizard 1984). Entrevistas han sido llevadas a cabo para averiguar el nivel de conocimientos que el público en general tiene sobre radiactividad y deshechos radiactivos en Gran Bretaña (Lucas 1987). Otros trabajos se han centrado en las represen- taciones conceptuales que los alumnos tienen sobre la radiactividad en el BUP (De Posada y Prieto 1989) y COU. (Prieto y de Posada 1988). En este trabajo que presentamos, estudiamos aspectos sociales sobre el tema, que de una $u$ otra forma se relacionan con los alumnos, haciendo éstos uso de los conocimientos cotidianos que puedan tener.

\section{MÉTODO DE TRABAJO}

Con la idea de que fuera representativo de la provincia, seleccionamos cuatro Institutos, dos estatales y dos privados, dos de Málaga capital y dos en pueblos de más de 50.000 habitantes. No hubo selección de alum. nos para realizar las pruebas, pasándolas a todos los componentes de las clases. Ninguno de los dos grupos habían estudiado la radiactividad. A veces se pasó el cuestionario en la hora de Ciencias, otras veces en horas de distintas asignaturas, pero siempre dentro del horario lectivo. La muestra estaba constituida por 334 alumnos, que desglosados por niveles, 206 eran de primero de BUP y 128 de tercero de Bachillerato de la rama de Ciencias. Atendiendo al sexo, contábamos con 168 chicas y 166 chicos. 


\section{PRIMERA PARTE DEL CUESTIONARIO}

Consistio en la presentación a los alumnos de los tres dibujos de la figura 1 , acompañados de la pregunta: ¿Donde está la radiactividad? Haz un círculo donde creas que está, si la hay. racion clínica, que probablemente contestan al azar. Las relaciones intergrupales carecen por tanto de signifícación a excepción de la expioración radiológica, con respuestas afirmativas más acusadas en el grupo de tercero.

Los alumnos que afirmaban que sí había radiactividad colocaban los círculos en lugares muy determinados,

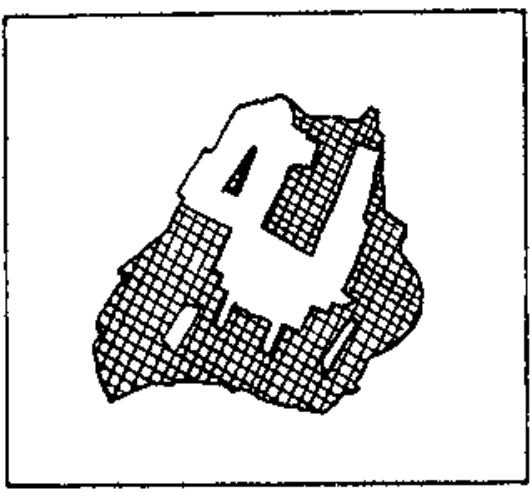

1

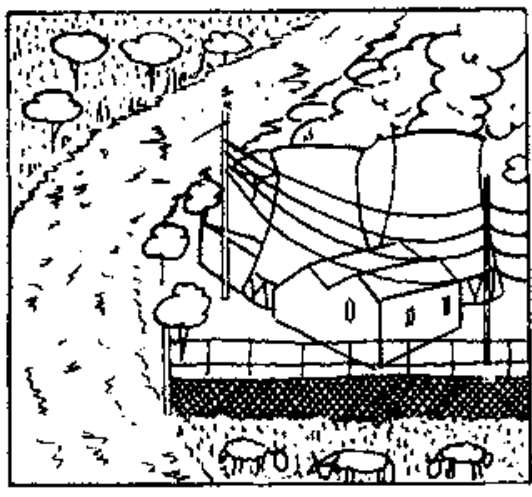

2

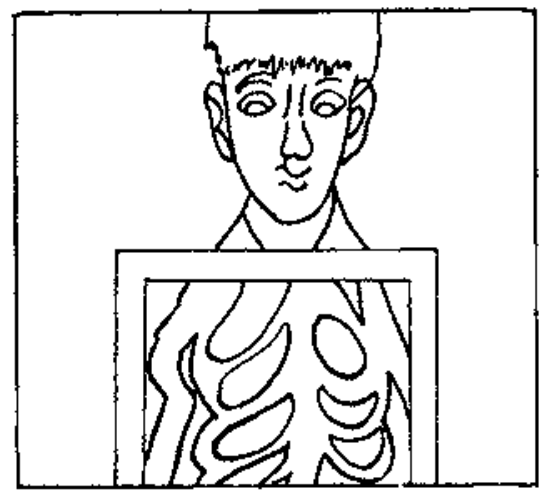

En el dibujo 1 puede observarse un trozo de mineral de Uranio, en el segundo podemos encontrar una central nuclear y su entorno próximo, en el tercer dibujo se observa una exploración radiologica de un paciente, algo con lo que todos nuestros alumnos de alguna forma están familiarizados. En la tabla 1 pueden encon * trarse las proporciones de respuestas afirmativas de ambos grupos de alumnos.

Tabla 1

Tantos por ciento de respuestas afirmativas en los dibujos de la figura 1 .

\begin{tabular}{|c|c|c|c|}
\hline Grupo & Mintral de uranio & Centre! nueleat & Expioracion $R \cdot X$ \\
\hline $1^{a}$ & 65 & 78 & 47 \\
\hline $3^{-\infty}$ & 66 & 71 & 68 \\
\hline
\end{tabular}

Se han estudiado los niveles de significación de estas respuestas con respecto a la frecuencia esperada si se hubiera emitido ésta al azar (Tabla 2), mostrándonos para el mineral de Uranio una significación $p<0.05$ para ambos grupos; en relación a la central nuclear $p<$ 0.01 también para ambos grupos; con respecto a la exploración con Rayos-X, $\mathrm{p}>0.05$ para los alumnos de primero, en cambio p $<0.01$ para el grupo de tercero.

Los alumnos opinan que en todos hay radiactividad, excepto los de primero de BUP en relación a la explo.
Tabla II

Tablas de contingencias por dibujo y por grupo. $f_{0}$ (frecuencia observada) $f_{c}$ (frecuencia esperada)

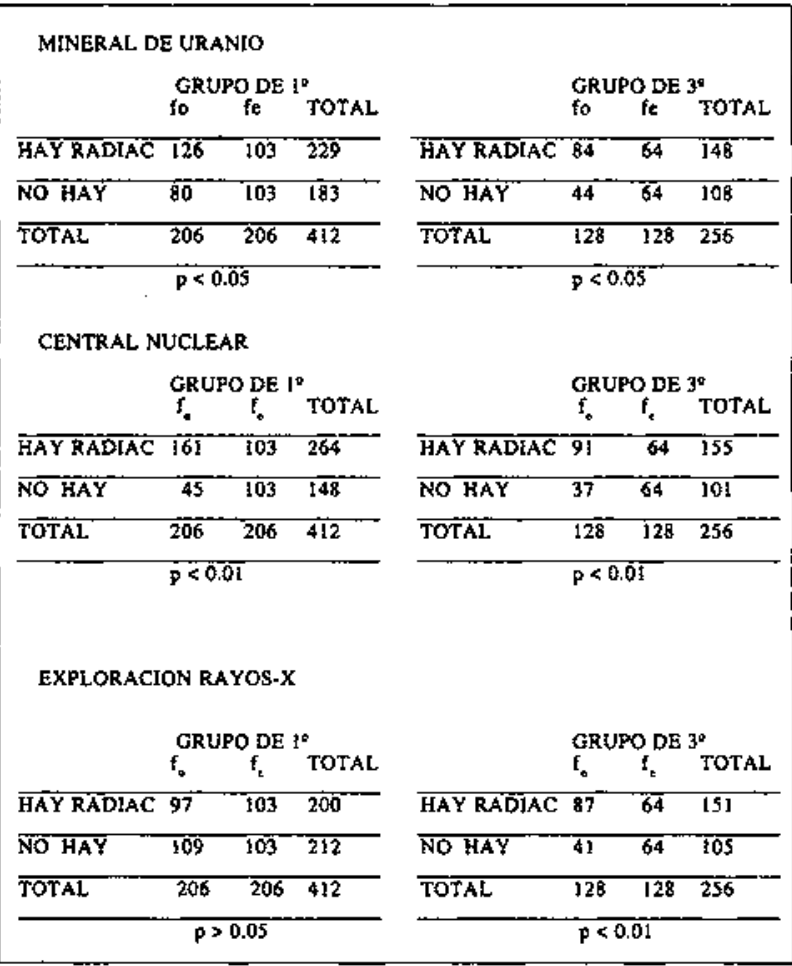

los tantos por ciento referidos a las posiciones, pueden encontrarse en la tabla 3 . 
Tabla III

Colocacion de los círculos en los dibujos de la figura 1.

\begin{tabular}{|c|c|}
\hline \multicolumn{2}{|c|}{ MINERAL DE URANIO } \\
\hline & 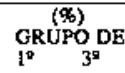 \\
\hline $\begin{array}{l}\text { El mineral } \\
\text { Minetel + aire } \\
\text { Centro mineral } \\
\text { Aire }\end{array}$ & $\begin{array}{l}52 \\
26 \\
17 \\
5\end{array}$ \\
\hline
\end{tabular}

EXPLORACION RAYOS $\mathrm{X}$

\begin{tabular}{lrr}
\hline & \multicolumn{2}{c}{ GRUPO DE } \\
& \multicolumn{1}{c}{$1^{\circ}$} & $3^{\circ}$ \\
\hline Todo et dibuja & 3 & 60 \\
Aparato & 24 & 25 \\
Paciente & 32 & 15 \\
Aire & 6 & 0 \\
\hline
\end{tabular}

CENTRAL NUCLEAR

\begin{tabular}{|c|c|}
\hline & 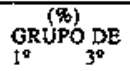 \\
\hline $\begin{array}{l}\text { Edificio +airededores } \\
\text { Todo ed dibujo } \\
\text { Núcieo } \\
\text { Humos } \\
\text { Elementos sueltos }\end{array}$ & $\begin{array}{rr}24 & 8 \\
26 & 73 \\
3 & 4 \\
8 & 4 \\
39 & 13\end{array}$ \\
\hline
\end{tabular}

Es de notar que los alumnos no tienen tanta certeza que haya radiactividad en el mineral de Uranio como en la central nuclear, como se pone de manifiesto por las diferencias en los tantos por ciento de las respuestas af irmativas de cada grupo entre sí, Ias razones que los alumnos aducen en este sentido serán expuestas más adelante. Para el mineral de Uranio la tendencia más común es la de hacer un círculo alrededor del mineral, pero también es relativamente frecuente introducir parte del aire que le rodea, suponiendo que éste también se contamina. Otra inclinación interesante es marcar alrededor del centro del mineral, según esta tendencia que ha sido detectada también en nuestras clases habituales, en las centrales nucleares se abre el mineral en dos, liberándose la energía que había encerrada en él. Esta tendencia es un tanto infantil, mal interpretando y confundiendo el núcleo del Uranio con el núcleo (centro) del mineral de Uranio.

Los alumnos relacionan principalmente radiactividad con centrales nucleares, como se pone de manifiesto en la tabla 2. Las tendencias mostradas en la central nuclear son variadas, la inclinación mayoritaria de tercero es la de marcar todo el dibujo, en cambio en primero se reparten las opiniones siendo mayoritarios los elementos sueltos. Es de destacar la baja inclinación en marcar el núcleo de la central, nuestros alumnos suponen que las operaciones se realizan en todo el edificio, saliendo fácilmente la radiactividad al exterior no sólo por los humos o las paredes, sino también por los cables eléctricos, como marcan en algunos dibujos. No obstante, las cifras obtenidas con el grupo de tercero creemos que han de tomarse con cierta reserva, nos parece excesiva la proporción de alumnos que marcan el dibujo completo, a pesar de que se les pidió que colocaran un círculo exactamente donde creían que había radiactividad. Este aspecto será aclarado en la sección de entrevistas.

En la tabla 3 aparecen las respuestas de primero a la exploración con Rayos- $X$, recordemos que no tiene demasiada validez ya que estadísticamente la hipótesis nula establecía en este caso, que los alumnos podían haber marcado al azar. Para tercero la inclinación mayoritaria es la de señalar todo el dibujo. Cuando se les pregunta a los alumnos en las entrevistas si hay o no radiactividad en la exploración radiológica, incluso los de tercero confiesan no conocer las razones que lo apoyan, pero afirman haber oído en los medios de comunicación esta tendencia, esto explicaría las discrepancias existentes entre ambos grupos $(p<0,01)$, ya que si suponemos un uso parecido de los medios de comunicación; los de tercero que ya han escogido la opción de Ciencias, prestan más atención a las noticias informativas de carácter científico que emiten los medios de comunicación de masas.

\section{SEGUNDA PARTE DEL CUESTIONARIO}

En esta segunda parte se propone una elección de términos bajo el siguiente enunciado:

De las siguientes palabras, señala tres con las que describas mejor la radiactividad: luz, ruido, fuego, curación, transformación, precaución, contaminación, calor.

Si hay alguna palabra que a tu juicio describa mejor la radiactividad, escribe hasta un máximo de dos.

Las palabras no se pueden considerar símiles, sino términos empleados en las explicaciones obtenidas en la prueba ya referida anteriormente (de Posada y Prieto 1989) y por tanto son vocablos relacionados con las ideas sobre radiactividad. Así lo entendieron los alumnos y aunque todas resultan ser elegidas (Tabla 4), hay una fuerte polarización en la opción $(p<0.01)$; desestimamos la hipótesis nula que apuntaba en la dirección de que los alumnos elegían tres términos al azar y adoptamos la hipótesis alternativa que apoya la idea que nuestros alumnos eligen palabras determinadas.

Efectivamente, son cuatro: contaminación, transformación, calor y precaución, las más escogidas en oruen decreciente para primero de BUP y transformación, contaminación, precaución y calor para tercero de BUP en igual orden. La palabra contaminación que era elegida por el $84 \%$ de los alumnos de primero pasa a ser elegida por el $68 \%$ de tercero, disminuyendo evidentemente su peso específico en el cómputo total; por otro lado las palabras precaución, transformación y curación aumentan ligeramente en detrimento de contaminacion, calor y otras menos elegidas.

Para los alumnos más jovenes el término contaminación es el más utilizado a la hora de referirse a la radiactividad, pero esta idea va evolucionando al admitir los alumnos de tercero el término transformación como más importante.

El número de palabras requeridas eran tres, pero los alumnos eligieron una media de 2.44 y.82 las chicas. $\mathrm{Se}$ estudiaron posibles diferencias significativas en la elección de términos con relación al sexo de los alum- 
Tabla IV

Restitados obteridos en la prueba de elección máltiple.

\begin{tabular}{|c|c|c|c|c|}
\hline & \multicolumn{2}{|c|}{$\begin{array}{c}\% \\
\text { sobre frases }\end{array}$} & \multicolumn{2}{|c|}{$\begin{array}{c}\% \\
\text { sobre alumnos }\end{array}$} \\
\hline & $1^{2}$ & $3^{2}$ & 10 & $3^{2}$ \\
\hline Luz & 4.5 & 3.5 & 12 & 9 \\
\hline Ruidos & 2 & 0 & $s$ & 0 \\
\hline Fuego & 2 & 1.5 & 6 & 4 \\
\hline Curación & 4 & 7 & 12 & 18 \\
\hline Transformación & 23 & 26 & 64 & 70 \\
\hline Precaucion & 17 & 21 & 46 & 55 \\
\hline Contaminacibn & 30.5 & 25.5 & 84 & 68 \\
\hline Cator & 17 & 15.5 & .47 & 42 \\
\hline
\end{tabular}

nos, no encontrándose correlación alguna $(p>0.05)$. Se les daba libertad para exponer otras palabras que definieran mejor la radiactividad, pero fueron muy pocos los que apuntaban otros términos, los chicos solían añadir: partículas, emisión, dañino, energía, fisión, indestructible, etc., y las chicas: destrucción, muerte, energía, etc. Aunque estadísticamente no tenga significación, los vocablos utilizados por los chicos son más técnicos pero también más impersonales, en cambio los utilizados por las chicas eran más mundanos, aunque más personales.

\section{ENTREVISTAS}

Para motivar a los alumnos a tratar más aspectos sobre el tema y poner sus conocimientos a prueba, hicimos uso de entrevistas utilizando los dibujos de la figura I cia previa en ellas, les mostraba uno a uno los dibujos de las figuras 1 y 2 , preguntándoles si había radiactividad o no, por qué y dónde creía que había, en caso afirmativo. Todas las entrevistas fueron transcritas posteriormente al papel.

Con el segundo dibujo de la figura 2, pretendíamos detectar diferencias percibidas por los alumnos en el comportamiento entre el mineral de Uranio y Uranio puro, pues anteriormente habíamos observado que un gran número de alumnos afirmaban que el mineral de Uranio no contiene radiactividad, y si la tiene es "natural" y no afecta al hombre; en cambio, el Uranio una vez elaborado, sí contiene radiactividad y ésta es muy peligrosa. Subyace a nuestro juicio una especie de prejuicio en el que nada que venga de la naturaleza puede ser malo o demasiado peligroso, diferenciando los alumnos más la procedencia natural o no del foco emisor, que la cantidad de radiactividad que ésta produzca. Los siguientes fragmentos apuntan en esa línea:

"La radiactividad se encuentra en el Uranio puro pero no en su mineral". "En el mineral hay un poco de radiactividad, pero es natural, en principio no perjudicial". "Es radiactivo, pero sólo es peligroso después de su utilización industrial".

El fragmento siguiente de entrevista puede ilustrar estas concepciones, en las que la mano del hombre parece estar detrás de todo el proceso:

E. - ¿Tendría radiactividad o no este mineral de Uranio?

A.- Un mineral de Uranio en sí no creo que transmita..., tendría que estar muy concentrado.

E.- ¿Por qué?
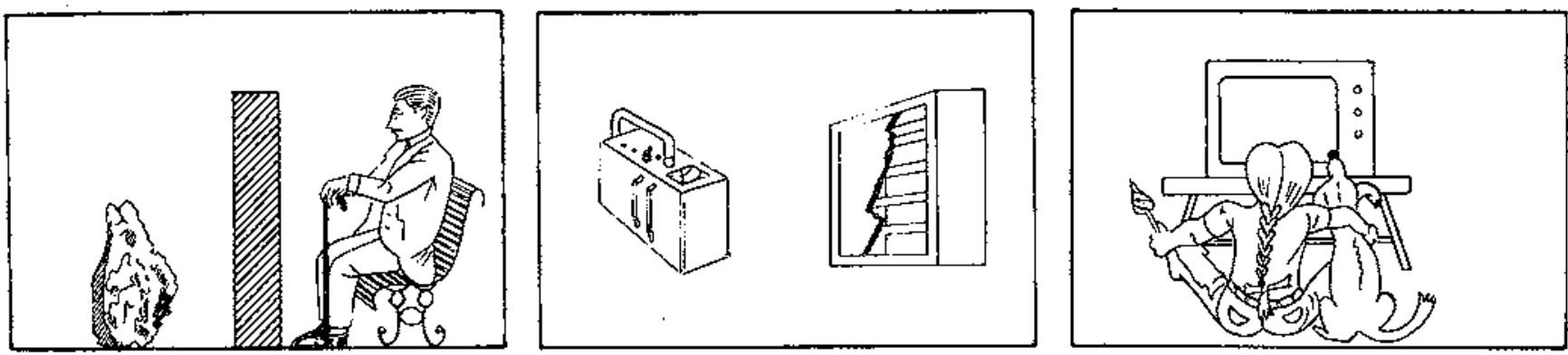

y 2. La técnica que seguimos es similar a la que describen Gilbert et al. (1982) llamada de aproximacion con ejemplos. (The interview about intances approach).

Se realizaron diez entrevistas, cinco de ellas individuales y cinco en grupos de dos alumnos; de éstos, diez eran de tercero de BUP rama de ciencias, y cinco de primero de bachillerato. Todas las entrevistas fueron realizadas por uno de los investigadores, con experien-
A.- Tiene que ser bombardeado.

E.- Por tanto el mineral de Uranio de la naturaleza, que contiene Uranio en cierta proporción, ¿será radiactivo o no?

A.- Yo creo que no. (Alumno de $3^{\circ}$ de BUP)

Con la central nuclear pretendíamos averiguar hasta que punto conocían los procesos que allí ocurren o cómo elios se los imaginan, la seguridad general de las 
instalaciones y el estado del medio ambiente exterior al recinto. Las ideas utilizadas por los alumnos de primero son cualitativamente parecidas a las de tercero de BUP, y éstas muy similares a las obtenidas por los alumnos de Física de COU (Prieto y de Posada 1988), después de haber visto este tema ampliamente en sus currículos (aproximadamente 30 horas de clases). Quizás las únicas diferencias estriban en que los de tercero y COU utilizan un vocabulario más técnico, reactor nuclear, índice de radiactividad, Uranio radiactivo, residuos radiactivos, etc. Los alumnos de primero entrevistados creen que hay radiactividad en todo el interior del edificio, en este sentido los alumnos de tercero concretizaron en el reactor nuclear (por este motivo nos parecen excesivos los datos obtenidos en la tabla 3, referentes a la localización de la radiactividad), aunque algunos de ellos opinan, al igual que otros de primero, que sólo se produce radiactividad cuando hay un escape, el fragmento siguiente lo ilustra:

"Creo que es porque se desprenden partículas nucleares que al entrar en contacto con la atmósfera producen radiactividad" (alumno de $1^{\text {o) }}$ ).

La explicación a este hecho encontrado es que la radiactividad sólo es noticia cuando se produce un escape, ciertos alumnos tienen conocimientos de la radiactividad cuando se produce una fuga en las centrales nucleares y los medios de comunicación se hacen eco de ello, confundiendo, estos alumnos, causas (el fenómeno de la radiactividad) y efectos producidos por la misma. La mayoría de los alumnos que opinan que hay radiactividad en el núcleo de la central, afirman que ésta no pasa al exterior (esta tendencia aumenta con la edad), mencionando fuertes medidas de seguridad, no obstante cierto número de alumnos opinan que la radiactividad sale fácilmente o con total libertad debido a los residuos radiactivos que pasan al río, los humos que se liberan a la atmósfera, o incluso por el tendido eléctrico que sale de la central nuclear.

Con la exploración radiológica y la televisión pretendíamos averiguar hasta qué punto consideran los alumnos que hay radiactividad en estos casos tan comunes para ellos y cuáles serían las causas para llevarles a tal afirmación. Es de notar que los alumnos de primero parecen responder al azar a la pregunta: ¿Hay o no radiactividad en la exploración radiológica? En cambio los de tercero afirman que sí la hay. Los datos obtenidos con las entrevistas en las que aparece la televisión son más bajos en respuestas afirmativas, pero la tendencia general es similar a la encontrada en la exploración radiológica. Hemos constatado cómo diferentes noticiarios pueden inducir este tipo de opiniones, al tratar al mismo tiempo temas de radiactividad y radiaciones producidas por aparatos de rayos- $X$ e incluso aparatos de televisión. Estos argumentos han sido ampliamente utilizados por los defensores de la energía nuclear en debates públicos televisados, y frases alusivas a la mayor peligrosidad de ver la televisión en color que vivir cerca de una central nuclear, son utilizadas con asiduidad.
Las ideas que los alumnos se hacen de los rayos- $\mathrm{X}$ son variadas, unos opinan que son "partículas que atraviesan nuestro cuerpo", "Los rayos-X son parecidos a los rayos B", "El aparato de rayos-X está basado en que las ondas o rayos radiactivos atraviesan el cuerpo sin dañarlo, proyectando una imagen interna de las partes duras (huesos)". La actividad radiactiva de los rayos-X a juicio de los alumnos es muy baja, aunque es acumulativa siendo peligroso a partir de cierto tiempo de exposición.

Con el primer dibujo de la figura 2 pretendíamos averiguar cómo se iban a comportar las concepciones de los alumnos sobre la radiactividad, al tener que explicar la interacción con un material sólido, como es el caso del muro, cuando se le preguntaba al científico si llegaría la radiactividad del mineral de Uranio. No se pretendian modificar conductas ni opiniones, sino poner en juego las que ya tienen. Los resultados obtenidos no son reproducibles, quizás porque los alumnos hubieran necesitado más tiempo para razonar y poner en orden sus concepciones antes de poder predecir cómo iba a ser la interacción entre el muro y la radiactividad.

\section{CONCLUSIONES}

En su mayor parte los alumnos creen que hay radiactividad en el mineral de Uranio, sin embargo, son muchos los que opinan que éste es natural y por tanto no es peligroso. Los alumnos están más convencidos de que hay radiactividad en las centrales nucleares que en el mineral de Uranio, Ias razones han sido expuestas. En relación a la exploración radiológica hemos detectado diferencias significativas intergrupales, esto parece apuntar en la dirección de que los alumnos de tercero que son de la opción de ciencias, aun utilizando los medios de comunicación en igual cuantía que los de primero, son más receptivos a noticias científicas, que dichos medios difunden periódicamente. Las entrevistas confirmaron de forma cualitativa los resultados obtenidos con las pruebas, dando para las entrevistas con el dibujo de la televisión resultados, en respuestas afirmativas, más bajos que con la exploración radiológica.

Como hemos podido constatar, los alumnos incluso los de primero tienen conocimientos sobre diversos aspectos de la radiactividad que hemos denominado sociales (su fuente de conocimiento es principalmente extraescolar), en contraposición a académicos; pues como hemos apuntado antes a estos alumnos no se les ha impartido aún la radiactividad en sus currículos. Estas ideas que les vienen de fuera, están mediatizadas por los constructos individuales que cada alumno pone en juego en la adquisición de conceptos y en la reestructuración de los mismos. 


\section{REFERENCIAS BIBLIOGRÁFICAS}

GILBERT, J.K., OSBORNE, R.J. y FENSHAN, P.J. 1982. Children'Science and its consequences for teaching, Science Education, pp.623-633.

LUCAS, A.M. 1987. Public Knowledge of radiation, Biologist, 34(3), pp. 125-129.

DE POSADA, J.M. y PRIETO, T. 1989. Ideas e interpretaciones de los alumnos sobre radiactividad, Revista de Educación, (en prensa).
PRIETO, T. y DE POSADA, J.M. 1988. Algunas ideas y errores conceptuales de los alumnos de COU sobre la radiactividad. V Congreso. Asociación Canaria para la enseñanza de las Ciencias.

SOLOMON, J. 1988. Una perspectiva social de los esquemas conceptuales, Investigación en la escuela, 5, pp. 17.22.

TIZARD, B. 1984. Problematic Aspects of Nuclear Education, Harvard Educational Review. 54(3), pp. 27I-281. 